\title{
Enhanced Reliability for Winding Machine via New Fault Tolerant Control Based on RST-Backstepping Controller
}

Fouad Haouari ${ }^{1, * 0}$, Salaheddine Messekher ${ }^{1}$, Noureddine Bali², Mohamed Tadjine ${ }^{3}$, Mohamed Seghir Boucherit ${ }^{3}$

${ }^{1}$ Laboratory of Innovative Technologies ENST Ex Biomédical, 16087 Bordj El Kiffan, Algiers, Algeria

${ }^{2}$ Electrical Engineering and Computing Faculty, USTHB, Algiers, Algeria

${ }^{3}$ Department of Electrical Engineering, Process Control Laboratory, ENP, Algiers, Algeria

Received: July 05, 2021; Accepted: October 03, 2021

\begin{abstract}
Due to the external disturbances, model uncertainties, strong coupling, and occurred faults, the winding machine presents a great control challenge. In order to deal with these problems, this paper presents the formulation of a novel scheme of fault tolerant control (FTC) for three-motor web-winding systems; it is concerned with the nonlinear robust backstepping control based on the combination of RST and backstepping controllers where the process is modelled by a nonlinear model. The main contribution of the paper is that the approach developed here summarises the performance of RST and backstepping controllers in order to design a robust controller capable of eliminating external disturbances and sensor faults affecting the system. The stability of the whole system is proven using the Lyapunov theory. Finally, analysis in comparison with the conventional backstepping controller and simulations in the MATLAB environment are accomplished to confirm the efficiency of the proposed method.
\end{abstract}

Keywords: winding machine $\bullet$ fault tolerant control • backstepping control $\bullet$ RST control

\section{Introduction}

The web-winding machine is used in many manufacturing systems, such as steel industries, processing paper, textiles, and film processes (Hou et al., 2016; Ponsart et al., 2010; Pagilla et al., 2007). The objective of a winding machine is to produce a strip with precise mechanical properties defined according to the tension web. The goal is to control the strip tension under the constraints of web velocity. Hence, the control of tension and speed of the continuous strip processing line is one of the most interesting applications needing high performance during system operation, where the components may encounter faults, such as uneven wear or fracture of the bearings and linkages, converter power loss and demagnetisation, and the open-circuit fault in an inverter leg, which can degrade the product quality and prevent continuous operation. Additionally, there are uncertainties and disturbances during the winding process (Hou et al., 2016). For this reason, it is highly motivating to use fault tolerant control (FTC) for improving system availability and reliability and reducing economic loss.

All the approaches to FTC can be categorised into two groups: active and passive (Elbakri and Boumhidi, 2020; Klimkowski, 2016; Medjmadj, 2019). The active approach requests a module for fault detection and isolation (FDI) in order to reconfigure the controller (Klimkowski, 2017). It has the capability to treat many types of faults. Nevertheless, it is sensitive to the result acquired from FDI and its design is hard for nonlinear systems with uncertainties. The passive approach is based on robust control. It does not require an FDI unit, and it has a fixed structure and parameters. It is fast enough to act rapidly in any irregular situation, but is proficient enough to accommodate only some faults established at the design stage.

In this respect, numerous FTC strategies have been proposed in the literature (Ponsart et al., 2010; Rodrigues et al., 2013; Xiao et al., 2011). The approaches are established based on the linear model suppositions of the system, such as the linear time invariant model (Xiao et al., 2011), the linear time variant model (Ponsart et al., 2010), and the linear 
parameter varying model (Rodrigues et al., 2013), where the nonlinear characteristics and coupling relationship between velocity and tension are not considered during the system modelling. In Ponsart et al. (2010), the authors established a FTC method for the nonlinear model of the system in order to decrease only the influence of the sensor fault.

To reply to the existing disadvantages and improve the performance of FTC in our research, we developed a new design of the FTC system for an unstable, non-linear, strong coupled, and multivariable system: a winding machine in the presence of sensor fault with uncertainty and disturbance. The objective of the proposed FTC system is to maintain current performance close to desirable performance and preserve stability conditions and robustness. The suggested approach is based on the combination of backstepping (Bodó and Lantos, 2019; Dashkovskiy and Pavlichkov, 2018; Haouari et al., 2020; Yu et al., 2018) and the RST controller (Ali et al., 2016; Brahim et al., 2017; Jiang et al., 2018; Khadraoui et al., 2014) to attain high performance against the occurred faults. The stability of the closed-loop system of the proposed controller is demonstrated based on the Lyapunov theory (Elmahfoud et al., 2020; Herizi and Barkat, 2019; Mohamed et al., 2020). Simulation results have shown the effectiveness and superiority of our proposed control law.

The remaining parts of the paper are organised as follows. Section 2 introduces the modelling of the winding machine. Section 3 describes concisely the RST algorithm for linear systems, while the developed FTC algorithm is described in Section 4. Simulation results are discussed in Section 5 and conclusions are drawn in Section 6.

\section{Modelling of the Winding Machine}

The winding process, presented in Figure 1, is composed of three reels driven by three motors, and the gear reduction is coupled with the reels. The three motors correspond to the unwinding reel, traction reel, and rewinding reel, respectively. The dynamic behaviour of a winding machine can be designated using a multivariable and nonlinear state space representation by the following equations (Chu et al., 2019).

$$
\left\{\begin{array}{l}
\frac{\mathrm{d} t_{1}}{\mathrm{~d} t}=\frac{E S_{a}}{L_{1}}\left(v_{2}-v_{1}\right)+\frac{1}{L_{1}} t_{0} v_{1}-\frac{1}{L_{1}} t_{1} v_{2} \\
\frac{\mathrm{d} v_{1}}{\mathrm{~d} t}=\frac{R_{1}^{2}}{J_{1}} t_{1}-\frac{b_{1}}{J_{1}} v_{1}-\frac{R_{1} n_{1}}{J_{1}} u_{1}-f_{c 1} v_{1}^{2} \\
\frac{\mathrm{d} v_{2}}{\mathrm{~d} t}=\frac{R_{2}^{2}}{J_{2}}\left(t_{2}-t_{1}\right)-\frac{b_{2}}{J_{2}} v_{2}+\frac{R_{2} n_{2}}{J_{2}} u_{2} \\
\frac{\mathrm{d} t_{2}}{\mathrm{~d} t}=\frac{E S_{a}}{L_{2}}\left(v_{3}-v_{2}\right)+\frac{1}{L_{2}} t_{1} v_{2}-\frac{1}{L_{2}} t_{2} v_{3} \\
\frac{\mathrm{d} v_{3}}{\mathrm{~d} t}=-\frac{R_{3}^{2}}{J_{3}} t_{2}-\frac{b_{3}}{J_{3}} v_{3}+\frac{R_{3} n_{3}}{J_{3}} u_{3}+f_{c 3} v_{3}^{2}
\end{array}\right.
$$

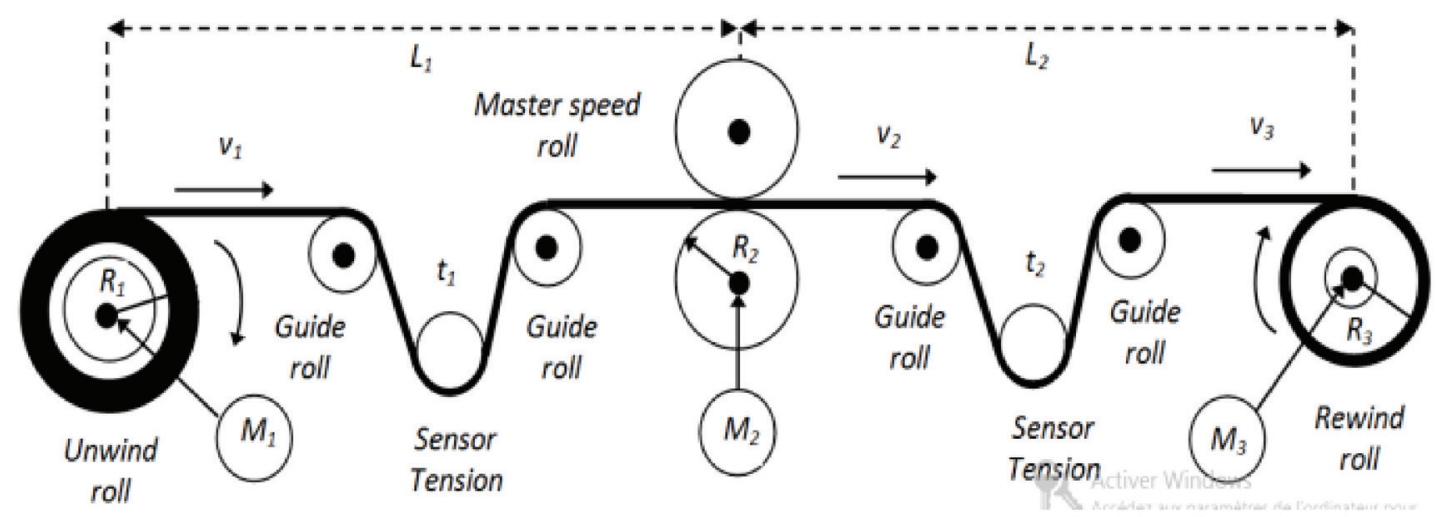

Fig. 1. Three-motor web-winding pilot system. 
with $f_{c i}=\left(\frac{h}{2 \pi J_{i}}\right)\left(\frac{J_{i}}{R_{i}^{2}}-2 \pi b \rho R_{i}^{2}\right)$ being a parameter that depends on the system characteristics and $i=1,2,3, \ldots \ldots$

The term $f_{c i}$ brings together the different parameters of the system. Thus, a change in the values of these parameters changes the value of $f_{c i}$ which is multiplied by the square of the speed, so this term has a considerable influence on the dynamics of the system.

Where $t_{1}$ and $t_{2}$ are web tensions $v_{1}, v_{2}$, and $v_{3}$ are web velocities, $\omega_{i}$ are angular velocities, $R_{i}$ are real-time radius of the $i^{\text {th }}$ reel, $L_{1}$ and $L_{2}$ are the web length for the first and second span, $t_{0}$ is wound-in web tension in the unwind roll, $u_{i}$ is input torque from the driving motor $M_{i}, b_{i}$ is friction coefficient of the $i^{\text {th }}$ roll shaft, $n_{i}$ is gearing ratio between the motor shaft and the corresponding roll shaft, $J_{i}$ is the effective inertia of the $i^{\text {it }}$ roll, $E$ is young's modulus, $S_{a}$ is cross-sectional area of the web, $\rho$ is density of the web, $b$ is width of the web, and $h$ is thickness of the web.

In the real situation of the web-winding system, there are present uncertainties in $R_{i}, J_{i}, E$, and $b_{i}$.

The variables, $t_{i}$ and $\omega_{i}$, can be measured via tensiometers and tachometers, and the variable $v_{i}$ can be acquired by $v_{i}=R_{i} \omega_{i}$; in that case, all the states are available by various sensors.

\section{Linear RST Controller Algorithm}

In a single-variable setting, the RST controller is a linear control device made of three polynomials $R(s), S(s)$, and $T(s)$ that must be definite in order to achieve efficient control; it is the controller with the maximum flexibility of application. The polynomials $R(s)$ and $S(s)$ tolerate the creation of a feedback control in order to reject disturbances and be robust to uncertainties; on the other hand, the polynomial $T(s)$ is added to the feed-forward to improve tracking. Figure 2 depicts the RST controller's overall construction, where $G(s)$ is the transfer function of the system, $r$ represents the reference input, $p_{s}$ is the disturbance, $y_{s}$ is the output signals, and $u_{s}$ is the control signals.

The RST controller is founded on the pole placement theory, which entails fixing polynomial $P(s)$ or, equally, their roots, i.e. the poles of the closed loop system, in advance. We are led to calculate polynomials $R(s)$ and $S(s)$ such that the Diophantine equation can be satisfied or similarly admits a solution if and only if $P(s)$ is a multiple of the greatest common divisor of $A(s)$ and $B(s)$; the controlled system's stability is verified if and only if the roots of the polynomial $P(s)$ and are all located in the left half-plane.

The output of the system is described as follows:

$$
y_{s}(s)=G(s) u_{s}(s)
$$

where $G(s)=B(s) / A(s)$ and $s$ is the Laplace variable.

The RST controller is specified by

$$
B(s) u_{\mathrm{s}}(s)=-S(s) y_{\mathrm{s}}(s)+T(s) r_{\mathrm{s}}(s)
$$

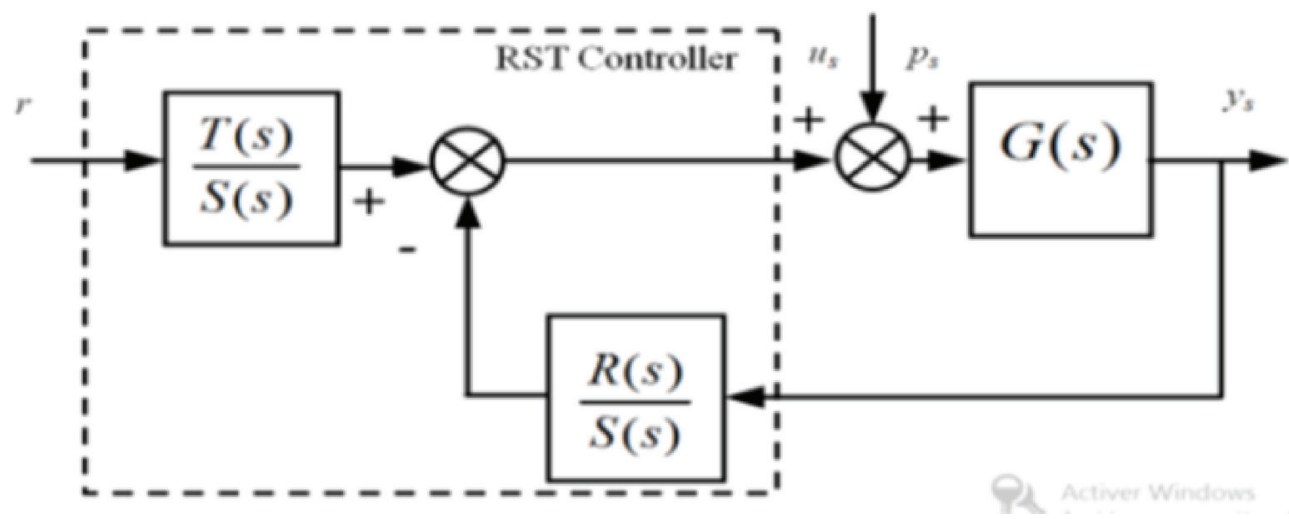

Fig. 2. Structure of RST controller. 
The polynomials $R(s), S(s)$, and $T(s)$ are specified by the following rules: The polynomials $R(s)$ and $S(s)$ are solutions of the Diophantine equation (Ali et al., 2016).

$$
P(s)=A(s) S(s)+B(s) R(s)
$$

where the roots of the polynomial $P(s)$ are established by means of the desired closed-loop poles.

The closed loop system is given by the following equation:

$$
y_{S}(\mathrm{~s})=\frac{B(s) T(s)}{P(s)} r_{s}(s)+\frac{S(s) B(s)}{P(s)} p_{s}(s)
$$

Let us denote by $\operatorname{deg}(P)$ the degree of the polynomial $P(s)$. The rejection of disturbance $p_{s}$ is confirmed by choosing $S(0)=0$.

The RST controller can guarantee a zero steady-state tracking performance error by taking the polynomial $T(s)$ constant and satisfy the condition $T(0)=R(0)$.

The polynomial $R, S$, and $T$ must verify the conditions: $\operatorname{deg}(P)=\operatorname{deg}(A)+\operatorname{deg}(S)$ and $\operatorname{deg}(S)=\operatorname{deg}(R)+1$.

As a result, $\operatorname{deg}(R) \geq \operatorname{deg}(A)$ and $\operatorname{deg}(A)=n_{a}$; at that point, we can conclude that

$$
\left\{\begin{array}{l}
\operatorname{deg}(S)=\operatorname{deg}(A)+1=n_{\mathrm{a}}+1 \\
\operatorname{deg}(R)=\operatorname{deg}(A)=n_{\mathrm{a}} \\
\operatorname{deg}(P)=2 n_{\mathrm{a}}+1
\end{array}\right.
$$

\section{RST-Backstepping Controller Synthesis}

The suggested FTC approach is based on a modified backstepping technique in which the stability is demonstrated in five steps utilising the Lyapunov theorem. This method enables high precision control and high-performance output tracking by ensuring that the error dynamics converge to zero asymptotically (Arsalan et al., 2018; Haouari et al., 2019; Karabacak and Eskikurt, 2011; Li et al., 2017). However, the backstepping-based controller fails to eliminate steady-state output errors due to parameters' uncertainties, disturbances, and faults. Then, in the final stage of backstepping control, when the polynomial coefficient gains are nonlinear, a nonlinear RST control action is introduced to assure high-precision control of the steady-state output. The primary idea behind the resultant method of RST-backstepping control is to construct a recursive controller in which the control laws are designed using state variables as virtual control signals, causing the stability of lower order subsystem. Finally, the real dynamic signal comes into the system loop controller; the suggested control system is designed step-by-step to improve the winding machine control performance. The schemas of the closed loop system using RST-backstepping are illustrated in Figure 3.

The state space model of the considered system can be formulated as follows:

$$
\left\{\begin{array}{l}
\dot{x}_{1}=f_{1}+a_{1} x_{2} \\
\dot{x}_{2}=f_{2}+a_{2} u_{1} \\
\dot{x}_{3}=f_{3}+a_{3} u_{2} \\
\dot{x}_{4}=f_{4}+a_{4} x_{5} \\
\dot{x}_{5}=f_{5}+a_{5} u_{3}
\end{array}\right.
$$

where $\left.x=\left[x_{1}, x_{2}, x_{3}, x_{4}, x_{5}\right]=t_{1}, v_{1}, v_{2}, t_{2}, v_{3}\right]$ and $f_{1}=\left(\frac{1}{L_{1}} t_{0}-\frac{E s}{L_{1}}\right) v_{1}, a_{2}=\frac{-R_{1} n_{1}}{J_{1}}, f_{2}=\frac{R_{1}^{2}}{J_{1}} t_{1}-\frac{b_{1}}{J_{1}} v_{1}-f_{c 1} v_{1}^{2}, f_{3}=\frac{R_{2}^{2}}{J_{2}}\left(t_{2}-t_{1}\right)-\frac{b_{2}}{J_{2}} v_{2}$, $a_{3}=\frac{R_{2} n_{2}}{J_{2}}, f_{4}=\left(\frac{1}{L_{2}} t_{1}-\frac{E s}{L_{2}}\right) v_{2}, f_{5}=-\frac{R_{3}^{2}}{J_{3}} t_{2}-\frac{b_{3}}{J_{3}} v_{3}+f_{c 3} v_{3}^{2}, a_{5}=\frac{R_{3} n_{3}}{J_{3}}, a_{1}=\frac{E s}{L_{1}}-\frac{1}{L_{1}} t_{1}, a_{4}=\frac{E s}{L_{2}}-\frac{1}{L_{2}} t_{2}$. 


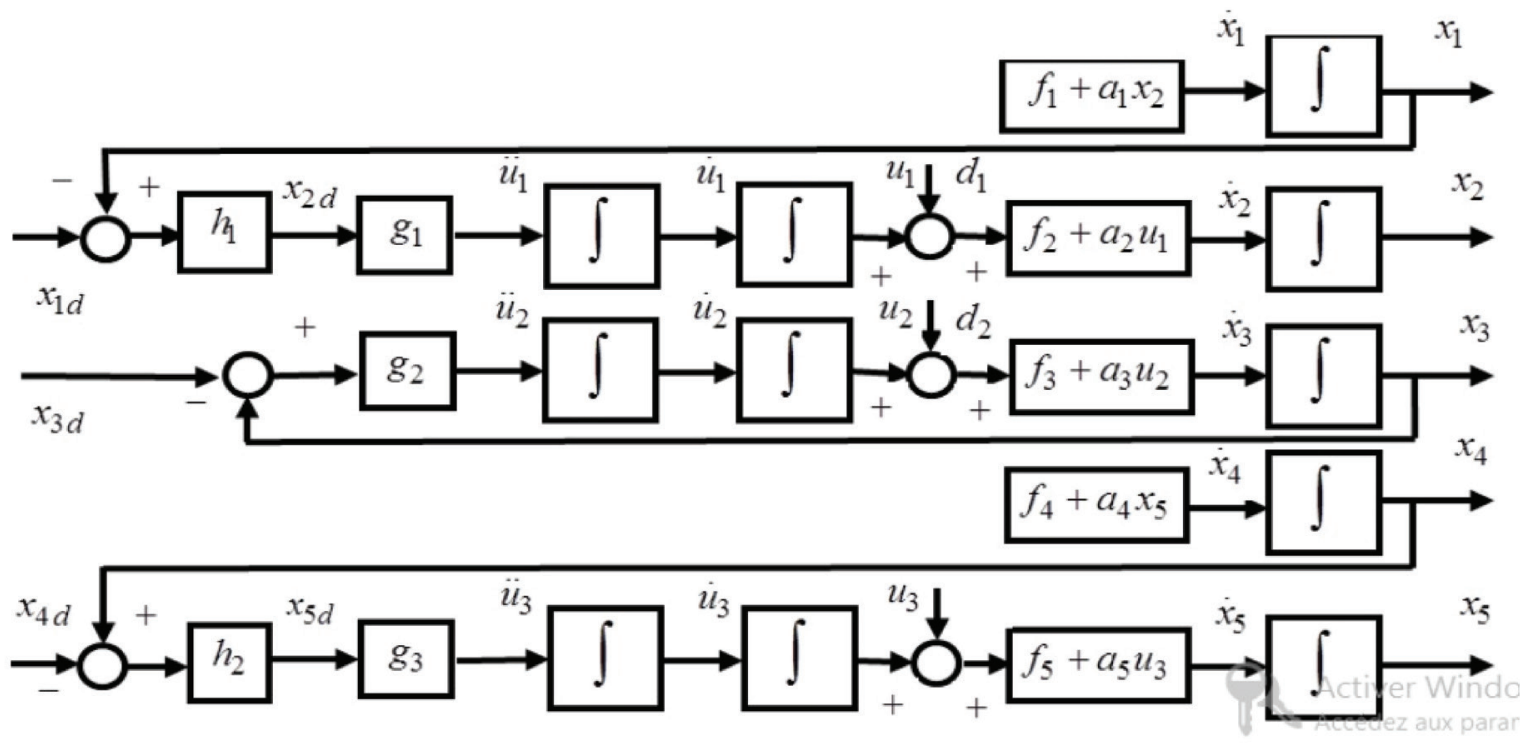

Fig. 3. Schemas of the closed loop system.

In the first step, we determine the virtual control that ensures the asymptotic convergence of the tracking error to zero (Chen et al., 2015; Haouari et al., 2018; Liu et al., 2018; Mohamed et al., 2020).

To proceed with the design, an error $e_{1}=x_{1}-x_{1 \mathrm{~d}}$ is described as the difference between the actual and the desired tension outputs. Its dynamic is given as

$$
\dot{e}_{1}=\dot{x}_{1}-\dot{x}_{1 \mathrm{~d}}=f_{1}+a_{1} x_{2}-\dot{x}_{1 \mathrm{~d}}
$$

Also, describing the second error $e_{2}=x_{2}-x_{2 d}$, we then obtain

$$
\dot{e}_{1}=f_{1}+a_{1}\left(e_{2}+x_{2 d}\right)-\dot{x}_{1 \mathrm{~d}}
$$

The error dynamics can be stabilised if the virtual control input $x_{2 d}$ is taken as follows:

$$
x_{2 d}=\frac{1}{a_{1}}\left(-f_{1}+\dot{x}_{1 \mathrm{~d}}-\lambda_{1} e_{1}\right)
$$

Then the dynamic of $e_{1}$ can be simplified into the following form:

$$
\dot{e}_{1}=-\lambda_{1} e_{1}+a_{1} e_{2}
$$

Let us confirm the tracking error stability by taking the Lyapunov candidate function $V_{1}=0.5 e_{1}^{2}$.

Its derivative is given as $\dot{V}_{1}=\dot{e}_{1} e_{1}$, at that time $\dot{V}_{1}=e_{1}\left(-\lambda_{1} e_{1}+a_{1} e_{2}\right)=-\lambda_{1} e_{1}^{2}+a_{1} e_{1} e_{2}$ where $\lambda_{1}$ must be a positive constant.

In the second step, a Lyapunov function, $V_{2}$, is established to guarantee convergence of the errors to zero, as follows: $V_{2}=V_{1}+0.5 e_{2}^{2}$.

Then consider the time derivative of the Lyapunov function $\dot{V}_{2}=-\lambda_{1} e_{1}^{2}+a_{1} e_{1} e_{2}+\dot{e}_{2} e_{2}$.

Since the main objective is to provide a robust performance with almost zero steady-state error, a concept of RST control action term is added (i.e., RST-backstepping strategy is adapted). 
In the case of linear RST control, with $S(s)=\alpha_{01}+\alpha_{11} s+\alpha_{12} s^{2}, R(s)=\beta_{01}+\beta_{11} s$ and $T(s)=\gamma_{01}+\gamma_{11} s+\gamma_{12} s^{2}$, the formula $\alpha_{01} u_{s}+\alpha_{11} \dot{u}_{s}+\alpha_{21} \ddot{u}_{s}=\gamma_{01} r_{s}+\gamma_{11} \dot{r}_{s}+\gamma_{21} \ddot{r}_{s}-\beta_{01} y_{s}-\beta_{11} \dot{y}_{s}$ represents the relationship between the output $y_{s}$, reference $r_{s}$, and the input $u_{s}$.

By applying the algorithm given in Section 3 , we can find $\alpha_{01}=0$ and $\gamma_{01}=\beta_{01}$, so the relationship between the input control $u$, output $y$ and reference $r$ can be written as

$$
\alpha_{11} \frac{\mathrm{d} u_{s}}{\mathrm{~d} t}+\alpha_{21} \frac{\mathrm{d}^{2} u_{s}}{\mathrm{~d} t^{2}}=\beta_{01} r_{s}+\gamma_{11} \dot{r}_{s}+\gamma_{21} \ddot{r}_{s}-\beta_{01} y_{s}-\beta_{11} \dot{y}_{s}
$$

However, in the situation of a nonlinear control by RST-backstepping, the only change in the last equation is that the constant coefficients $\alpha_{12}, \alpha_{11}, Y_{11}, \gamma_{21}, \beta_{01}$, and $\beta_{11}$ become nonlinear function gains depend on the system nonlinearity and also by applying this to the subsystem $\dot{x}_{2}=f_{2}+a_{2} u_{1}$, by using the corresponding variables, the input $u_{1}$, the state $x_{2}$ which is correspond to the output of the subsystem and the reference $x_{2 d}$, one gets that input control $u_{1}$ can be defined as

$$
\alpha_{11} \frac{\mathrm{d} u_{1}}{\mathrm{~d} t}+\alpha_{21} \frac{\mathrm{d}^{2} u_{1}}{\mathrm{~d} t^{2}}=\beta_{01} x_{2 d}+\gamma_{11} \dot{x}_{2 d}+\gamma_{21} \ddot{x}_{2 d}-\beta_{01} x_{2}-\beta_{11} \dot{x}_{2}
$$

In order to find a solution for proving the stability of the closed loop system and the negativity of the Lyapunov function, it is necessary to neglect some gains and fix other gains.

Then, putting $\mathrm{V}_{21}=0$ and using from Eq. (6) the first and second derivative of $u_{1}$ with Eq. (13) gives

$$
\left(\alpha_{11} / a_{2}\right)\left(\ddot{e}_{2}+\ddot{x}_{2 d}-\dot{f}_{2}\right)+\left(\alpha_{21} / a_{2}\right)\left(\ddot{e}_{2}+\dddot{x}_{2 \mathrm{~d}}-\ddot{f}_{2}\right)=-\beta_{01} e_{2}-\beta_{11} \dot{e}_{2}
$$

In the case of fixing $\alpha_{11}=0$ and $\alpha_{21}=a_{2}$, the derivative of the errors $e_{2}$ is obtained; then, integrating it twice, with respect to time, gives

$$
\dot{e}_{2}+\dot{x}_{2 \mathrm{~d}}-f_{2}=-\beta_{01} \int_{0}^{t}\left(\int_{0}^{t} e_{2} \mathrm{~d} \rho\right) \mathrm{d} \rho-\beta_{11} \int_{0}^{t} e_{2} \mathrm{~d} \rho
$$

Then

$$
\dot{e}_{2}=f_{2}-\dot{x}_{2 \mathrm{~d}}-\beta_{01} \int_{0}^{t}\left(\int_{0}^{t} e_{2} \mathrm{~d} \rho\right) \mathrm{d} \rho-\beta_{11} \int_{0}^{t} e_{2} \mathrm{~d} \rho
$$

One may get

$$
\dot{V}_{2}=-\lambda_{1} e_{1}^{2}+e_{2}\left(a_{1} e_{1}+f_{2}-\dot{x}_{2 \mathrm{~d}}-\beta_{01} \int_{0}^{t}\left(\int_{0}^{t} e_{2} \mathrm{~d} \rho\right) \mathrm{d} \rho-\beta_{11} \int_{0}^{t} e_{2} \mathrm{~d} \rho\right)
$$

In order to guarantee that the derivative of the Lyapunov function is negative, the parameter $\beta_{01}$, in addition to $\beta_{11}$, must be chosen as $\beta_{01}=\delta_{01} \operatorname{sgn}\left(z_{01}\right)$ and $\beta_{11}=\delta_{11} \operatorname{sgn}\left(z_{11}\right)$, where $z_{01}=e_{2} \int_{0}^{t} e_{2}(\rho) d \rho$ and $z_{11}=e_{2} \int_{0}^{t}\left(\int_{0}^{t} e_{2} \mathrm{~d} \rho\right) d \rho$, and where the gains $\delta_{01}$ is selected such that

$$
\left|\delta_{01} \operatorname{sgn}\left(z_{01}\right) \int_{0}^{t} e_{2}(\rho) \mathrm{d} \rho\right|>\Delta_{1}
$$

with $\Delta_{1} \geq\left|a_{1} e_{1}\right|+\left|f_{2}(x)-\dot{x}_{\mathrm{d} 2}\right|$.

At that time,

$$
\dot{V}_{2}=-\lambda_{1} e_{1}^{2}-\beta_{01} e_{2} \int_{0}^{t}\left(\int_{0}^{t} e_{2} \mathrm{~d} \rho\right) \mathrm{d} \rho+e_{2}\left(a_{1} e_{1}+f_{2}-\dot{x}_{2 \mathrm{~d}}-\beta_{11} \int_{0}^{t} e_{2} \mathrm{~d} \rho\right)
$$


As a result $\dot{V}_{2} \leq 0$.

In the third step, defining the third Lyapunov function as $V_{3}=V_{2}+0.5 e_{3}^{2}$, the derivation of $V_{3}$ is then obtained as

$$
\dot{V}_{3}=\dot{V}_{2}+\dot{e}_{3} e_{3}
$$

Defining the error $e_{3}$ as follows $e_{3}=x_{3}-x_{3 \mathrm{~d}}$, we obtain its time derivative as $\dot{e}_{3}=f_{3}+a_{3} u_{2}-\dot{x}_{3 \mathrm{~d}}$.

Using the formula specified by Eq. (12) with corresponding variables $u_{2}, x_{3}$, and $x_{3 d}$, the input control $u_{2}$ can be formulated as

$$
\alpha_{12} \frac{\mathrm{d} u_{2}}{\mathrm{~d} t}+\alpha_{22} \frac{\mathrm{d}^{2} u_{2}}{\mathrm{~d} t^{2}}=\beta_{02} x_{3 d}+\gamma_{12} \dot{x}_{3 d}+\gamma_{22} \ddot{x}_{3 d}-\beta_{02} x_{3}-\beta_{12} \dot{x}_{3}
$$

Taking $\mathrm{Y}_{22}=0$ and substituting the first and second derivative of $u_{2}$ extracted from Eq. (6) in Eq. (21), we obtain

$$
\left(\alpha_{12} / a_{3}\right)\left(\ddot{e}_{3}+\ddot{x}_{3 d}-\dot{f}_{3}\right)+\left(\alpha_{22} / a_{3}\right)\left(\dddot{e}_{3}+\dddot{x}_{3 \mathrm{~d}}-\ddot{f}_{3}\right)=-\beta_{02} e_{3}-\beta_{12} \dot{e}_{3}
$$

Putting $\alpha_{12}=0$ and $\alpha_{22}=a_{3}$, the derivative of the errors $e_{3}$ is obtained; then, integrating $\dot{e}_{3}$ twice, with respect to time, gives

$$
\dot{e}_{3}+\dot{x}_{3 \mathrm{~d}}-f_{3}=-\beta_{03} \int_{0}^{t}\left(\int_{0}^{t} e_{3} \mathrm{~d} \rho\right) \mathrm{d} \rho-\beta_{13} \int_{0}^{t} e_{3} \mathrm{~d} \rho
$$

In that case

$$
\dot{e}_{3}=f_{3}-\dot{x}_{3 \mathrm{~d}}-\beta_{02} \int_{0}^{t}\left(\int_{0}^{t} e_{3} \mathrm{~d} \rho\right) \mathrm{d} \rho-\beta_{12} \int_{0}^{t} e_{3} \mathrm{~d} \rho
$$

Thus, we can get $\dot{V}_{3}$ as follows

$$
\dot{V}_{3}=\dot{V}_{2}+e_{3}\left(f_{3}-\dot{x}_{3 \mathrm{~d}}-\beta_{02} \int_{0}^{t}\left(\int_{0}^{t} e_{3} \mathrm{~d} \rho\right) \mathrm{d} \rho-\beta_{12} \int_{0}^{t} e_{3} \mathrm{~d} \rho\right)
$$

To make $\dot{V}_{3}$ negative, the parameters $\beta_{02}$ and $\beta_{12}$ can be taken as $\beta_{02}=\delta_{02} \operatorname{sgn}\left(\mathrm{z}_{02}\right)$ and $\beta_{12}=\delta_{12} \operatorname{sgn}\left(z_{12}\right)$, with $z_{02}=e_{3} \int_{0}^{t} e_{3}(\rho) d \rho$ and $z_{12}=e_{3} \int_{0}^{t}\left(\int_{0}^{t} e_{3} \mathrm{~d} \rho\right) d \rho$.

Further, supposing that the gain $\delta_{02}$ is selected such that

$$
\left|\delta_{02} \operatorname{sgn}\left(z_{02}\right) \int_{0}^{t} e_{3}(\rho) \mathrm{d} \rho\right|>\Delta_{2}
$$

with $\Delta_{2} \geq\left|f_{3}(x)-\dot{x}_{\mathrm{d} 3}\right|$, the resultant equation can be obtained as

$$
\dot{V}_{3}=\dot{V}_{2}-\beta_{02} e_{3} \int_{0}^{t}\left(\int_{0}^{t} e_{3} \mathrm{~d} \rho\right) \mathrm{d} \rho+e_{3}\left(f_{3}-\dot{x}_{3 \mathrm{~d}}-\beta_{12} \int_{0}^{t} e_{2} \mathrm{~d} \rho\right)
$$

After that, it is concluded that $\dot{V}_{3} \leq 0$.

In the fourth step, the Lyapunov function $V_{4}$ is defined as

$$
V_{4}=V_{3}+0.5 e_{4}^{2}
$$


taking the derivation of $V_{4}$ as $\dot{V}_{4}=\dot{V}_{3}+\dot{e}_{4} e_{4}$, where $e_{4}=x_{4}-x_{4 \mathrm{~d}}$; then $\dot{e}_{4}=f_{4}+a_{4} x_{5}-\dot{x}_{4 \mathrm{~d}}$, so the derivative of $V_{4}$ is

$$
\dot{V}_{4}=\dot{V}_{3}+e_{4}\left(f_{4}+a_{4} x_{5}-\dot{x}_{4 \mathrm{~d}}\right)
$$

where $e_{5}=x_{5}-x_{5 \mathrm{~d}}$; at that time, the resulting derivatives of $V_{4}$ are given as $\dot{V}_{4}=\dot{V}_{3}+e_{4}\left(f_{4}+a_{4}\left(e_{5}+x_{5 \mathrm{~d}}\right)-\dot{x}_{4 \mathrm{~d}}\right)$.

Then, choosing

$$
x_{5 \mathrm{~d}}=\frac{1}{a_{4}}\left(-f_{4}+\dot{x}_{4 d}-\lambda_{2} e_{4}\right)
$$

we subsequently obtain $\dot{V}_{4}=\dot{V}_{3}-\lambda_{2} e_{4}^{2}+a_{4} e_{4} e_{5}$.

Finally, in the fifth step, consider the Lyapunov function $V_{5}$ as follows $V_{5}=V_{4}+0.5 e_{5}^{2}$. Its time derivative is given as $\dot{V}_{5}=\dot{V}_{4}+e_{4} \dot{e}_{4}$.

By means of Eq. (12) and the assignable variables $u_{3}, x_{5}$ and $x_{5 d^{\prime}}$, the input control $u_{3}$ can be expressed as

$$
\alpha_{13} \frac{\mathrm{d} u_{3}}{\mathrm{~d} t}+\alpha_{23} \frac{\mathrm{d}^{2} u_{3}}{\mathrm{~d} t^{2}}=\beta_{03} x_{5 d}+\gamma_{13} \dot{x}_{5 d}+\gamma_{23} \ddot{x}_{5 d}-\beta_{03} x_{5}-\beta_{13} \dot{x}_{5}
$$

By putting $\mathrm{Y}_{23}=0$ and from Eq. (6) utilising the first and second derivatives of $u_{3}$, exploiting it in Eq. (31) yields

$$
\left(\alpha_{13} / a_{5}\right)\left(\ddot{e}_{5}+\ddot{x}_{5 d}-\dot{f}_{5}\right)+\left(\alpha_{23} / a_{5}\right)\left(\ddot{e}_{5}+\dddot{x}_{5 \mathrm{~d}}-\ddot{f}_{5}\right)=-\beta_{03} e_{5}-\beta_{13} \dot{e}_{5}
$$

It is required to choose $\alpha_{13}=0$ and $\alpha_{23}=a_{5}$, to find the derivative of $e_{5}$; subsequently, integrating it twice yields

$$
\dot{e}_{5}+\dot{x}_{5 \mathrm{~d}}-f_{5}=-\beta_{03} \int_{0}^{t}\left(\int_{0}^{t} e_{5} \mathrm{~d} \rho\right) \mathrm{d} \rho-\beta_{13} \int_{0}^{t} e_{5} \mathrm{~d} \rho
$$

At that moment

$$
\dot{e}_{5}=f_{5}-\dot{x}_{5 \mathrm{~d}}-\beta_{03} \int_{0}^{t}\left(\int_{0}^{t} e_{5} \mathrm{~d} \rho\right) \mathrm{d} \rho-\beta_{13} \int_{0}^{t} e_{5} \mathrm{~d} \rho
$$

Subsequently, $\dot{V}_{5}$ become

$$
\dot{V}_{5}=\dot{V}_{4}+e_{5}\left(a_{4} e_{4}+f_{5}-\dot{x}_{5 \mathrm{~d}}-\beta_{03} \int_{0}^{t}\left(\int_{0}^{t} e_{5} \mathrm{~d} \rho\right) \mathrm{d} \rho-\beta_{13} \int_{0}^{t} e_{5} \mathrm{~d} \rho\right)
$$

Consider taking $\beta_{03}=\delta_{03} \operatorname{sgn}\left(z_{03}\right)$ and $\beta_{13}=\delta_{13} \operatorname{sgn}\left(z_{13}\right)$ with $z_{03}=e_{5} \int_{0}^{t} e_{5}(\rho) d \rho$ and $z_{13}=e_{5} \int_{0}^{t}\left(\int_{0}^{t} e_{5} \mathrm{~d} \rho\right) d \rho$, where the gain $\delta_{03}$ is selected such that

$$
\left|\delta_{03} \operatorname{sgn}\left(z_{03}\right) \int e_{5}(\rho) \mathrm{d} \rho\right|>\Delta_{3}
$$

where $\Delta_{3} \geq\left|a_{4} e_{4}\right|+\left|f_{5}(x)-\dot{x}_{d 5}\right|$. In that case

$$
\dot{V}_{5}=\dot{V}_{4}-\beta_{03} e_{5} \int_{0}^{t}\left(\int_{0}^{t} e_{5} \mathrm{~d} \rho\right) \mathrm{d} \rho+e_{5}\left(a_{4} e_{4}+f_{5}-\dot{x}_{5 \mathrm{~d}}-\beta_{13} \int_{0}^{t} e_{5} \mathrm{~d} \rho\right)
$$


Consequently, $\dot{V}_{5} \leq 0$; and this allows us to conclude that all the signals in the system are asymptotically stable. where the nonlinear functions are as follows:

$$
\begin{aligned}
& g_{1}=\left(1 / \alpha_{21}\right)\left(-\alpha_{11} \dot{u}_{1}+\beta_{01} x_{2 d}+\gamma_{11} \dot{x}_{2 d}+\gamma_{21} \ddot{x}_{2 d}-\beta_{01} x_{2}-\beta_{11} \dot{x}_{2}\right) \quad h_{1}=\left(1 / a_{1}\right)\left(-f_{1}+\dot{x}_{1 d}-\lambda_{1} e_{1}\right) \\
& g_{2}=\left(1 / \alpha_{22}\right)\left(-\alpha_{12} \dot{u}_{2}+\beta_{02} x_{3 d}+\gamma_{12} \dot{x}_{3 d}+\gamma_{22} \ddot{x}_{3 d}-\beta_{02} x_{3}-\beta_{12} \dot{x}_{3}\right) \\
& g_{3}=\left(1 / \alpha_{23}\right)\left(-\alpha_{13} \dot{u}_{3}+\beta_{03} x_{5 d}+\gamma_{13} \dot{x}_{5 d}+\gamma_{23} \ddot{x}_{5 d}-\beta_{03} x_{5}-\beta_{13} \dot{x}_{5}\right) \quad h_{2}=\left(1 / a_{4}\right)\left(-f_{4}+\dot{x}_{4 d}-\lambda_{2} e_{4}\right)
\end{aligned}
$$

\section{Simulation Results and Analysis}

In this section, the effectiveness of the proposed FTC method is confirmed through simulation.

The winding machine structural parameters are taken as:

$t_{0}=2 \mathrm{~N}, \rho=800 \mathrm{~kg} / \mathrm{m}^{3}, b=0.1 \mathrm{~m}, h=1.2 \times 10^{-4} \mathrm{~m}, 0.018 \mathrm{~m} \leq R_{1} \leq 0.1 \mathrm{~m}, R_{2}=0.02 \mathrm{~m}, 0.018 \mathrm{~m} \leq R_{3} \leq 0.1 \mathrm{~m}$,

$b_{1}=5 \times 10^{-3} \mathrm{Nm} / \mathrm{rad} / \mathrm{s}, E=1.5 \times 10^{7} \mathrm{~N} / \mathrm{m}^{2}, L_{1}=L_{2}=1.2 \mathrm{~m}, J_{1}=J_{3}=6.76 \times 10^{-6} \mathrm{~kg} / \mathrm{m}^{2}, n_{1}=1, J_{2}=8.67 \times 10^{-4} \mathrm{~kg} / \mathrm{m}^{2}$,

$b_{2}=6.5 \times 10^{-3} \mathrm{Nm} / \mathrm{rad} / \mathrm{s}, n_{2}=1, b_{3}=4.6 \times 10^{-3} \mathrm{Nm} / \mathrm{rad} / \mathrm{s}, n_{3}=1$.

It is assumed that the desired outputs of web tensions are $10 \mathrm{~N}$ and the web velocity is $1 \mathrm{rad} / \mathrm{s}$.

Our objective is to roll up the strip in an accurate way. This can be attained if the tensions $t_{1}$ and $t_{2}$ and the velocity $v_{2}$ are mainly kept to their reference inputs.

In order to illustrate that the proposed method can deal with various faults, the following two cases, the case of disturbance, and after that the case of sensor fault and uncertainty, are considered.

The improved performance given by the proposed controller can be shown by using an index / comprising the integral of square errors. It is given as follows.

$$
I=\int_{0}^{\infty} \mathrm{e}_{1}(\mathrm{t})^{2} \mathrm{dt}+\int_{0}^{\infty} \mathrm{e}_{3}(\mathrm{t})^{2} \mathrm{dt}+\int_{0}^{\infty} \mathrm{e}_{4}(\mathrm{t})^{2} \mathrm{dt}
$$

\subsection{First scenario: External disturbance}

The goal of this scenario is to evaluate the winding machine performance. In this situation, external disturbances, which represent all external torque which increase or decrease the motors torque, are added at $t=40 \mathrm{~s}$ to the inputs of the first motor, and it is considered periodic $d_{1}=2 \times \cos (2 \times \mathrm{t})$.

From Figure 4 and Table 1, by comparing the proposed controller and the conventional controller, it can be seen that the proposed controller gives the best performance, can compensate the effects of external disturbance, and can guarantee the asymptotic stability of the closed-loop system with good transient performance. It can be attained after a short settling time, with minor steady-state errors; in addition, it can make the tension and velocity track their desired trajectories with tolerable amplitude of control inputs, when the web-winding system suffers from disturbances.

\subsection{Second scenario: Sensor faults and uncertainty}

In this situation, an uncertainty at $t=40 \mathrm{~s}$ is added to the system in the moment of inertia $J_{1}$ of the first roll, with $40 \%$ of the nominal value due to factors related to the structure of the winding machine and the following abrupt fault $f_{s}$ which affects the second sensor:

$$
f_{s}=\left\{\begin{array}{lc}
1 & 40 \mathrm{~s}<t \leq 80 \mathrm{~s} \\
0 & 0 \mathrm{~s}<t \leq 40 \mathrm{~s}
\end{array}\right.
$$

When a sensor fault occurs, the faulty measurements directly corrupt the closed-loop behaviour. Moreover, the controller aims at cancelling the error between the measurement and its reference input. Figure 5 displays the system output tracking results. Evidently, the proposed FTC approach has the best performance under sensor fault and uncertainty. From the simulation results, it is apparent that the settling time is shortened and the control inputs are in the admissible amplitude. In contrast, the traditional approach is poor in performance. It is characterised by great settling time and large steady state errors. 


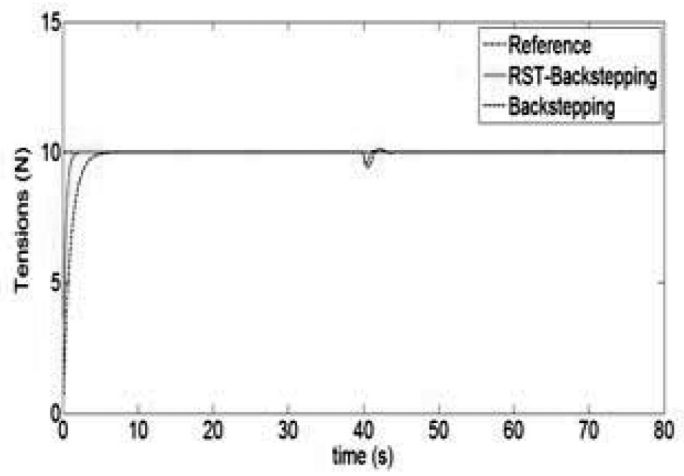

(a) Tensions $t_{1}$

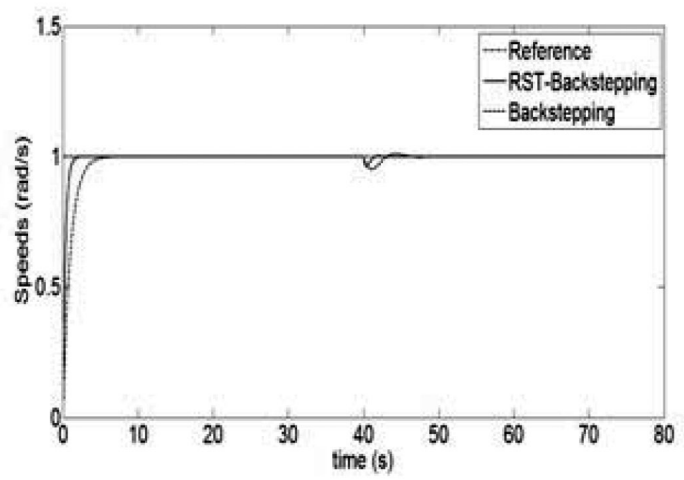

(c) Speeds $v_{2}$

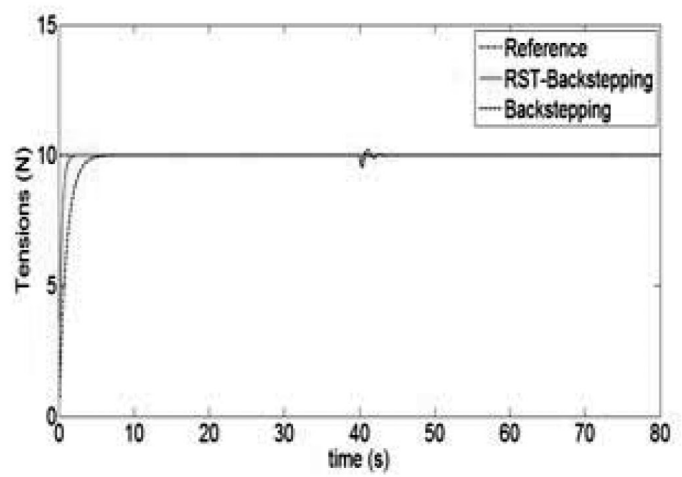

(e) Tensions $t_{2}$

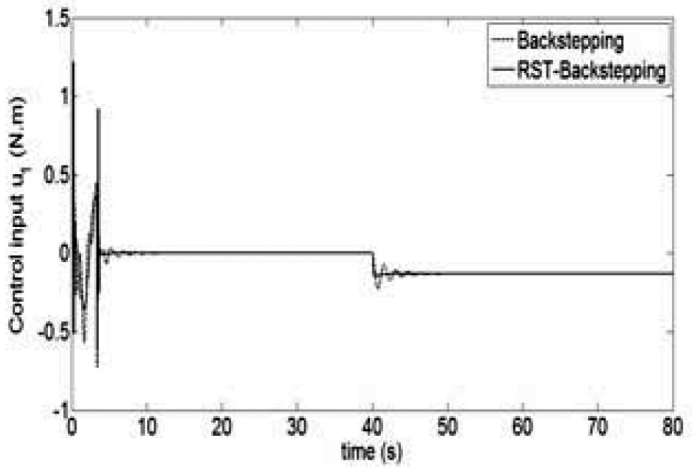

(b) Control inputs $u_{1}$

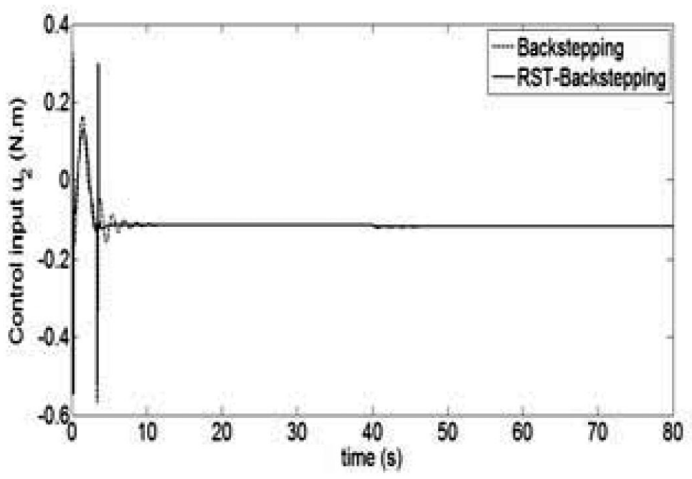

(d) Control inputs $u_{2}$

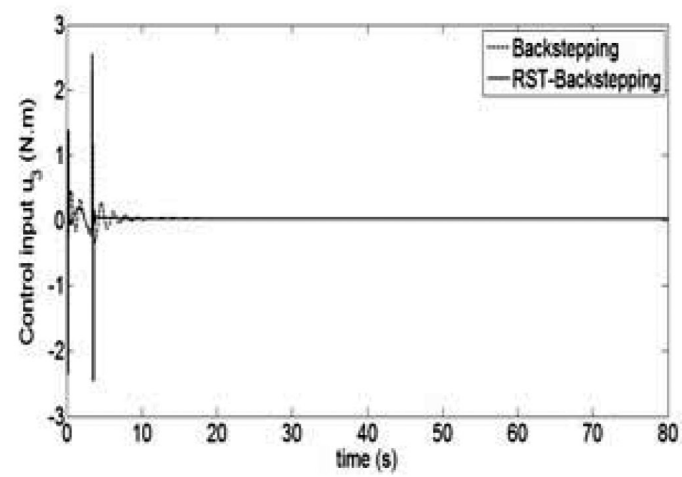

(f) Control inputs $u_{3}$

Fig. 4. Performance evaluation under the first scenario. (a) web tensions $t_{1}$, (b) control inputs $u_{1}$, (c) web velocities $V_{2}$, (d) control inputs $u_{2}$, (e) web tensions $t_{2}$, (f) control inputs $u_{3}$.

Table 1. The performance comparison.

\begin{tabular}{lll}
\hline Parameters & Backstepping & RST-Backstepping \\
\hline \hline$\lambda=\left[\lambda_{1}, \lambda_{2}\right]$ & & $\lambda=[100,20]$ \\
$\delta_{0}=\left[\delta_{01}, \delta_{02}, \delta_{03}\right]$ & $\lambda=[100,20]$ & $\delta_{0}=[300,260,210]$ \\
$\delta_{1}=\left[\delta_{11}, \delta_{12}, \delta_{13}\right]$ & & $\delta_{1}=[316,267,213]$ \\
$t_{s}(s)=\left[t_{s 1}, t_{s 2}, t_{s 3}\right]$ & $t_{s}=[2.94,3.01,3.1]$ & $t_{s}=[1,1.1,1.14]$ \\
$l$ & 98.43 & 30.01 \\
\hline
\end{tabular}




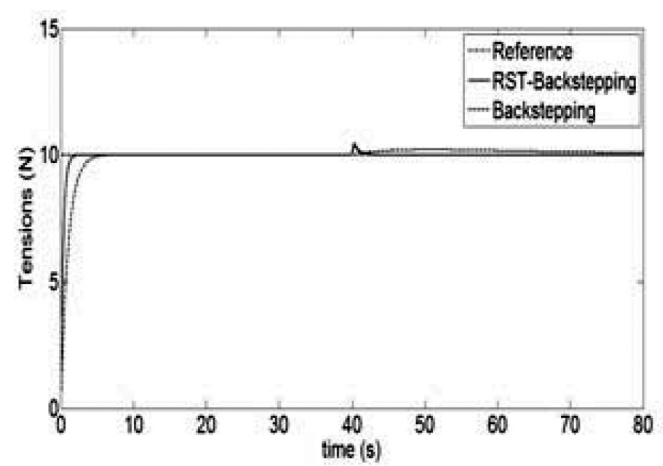

(a) Tensions $t_{1}$

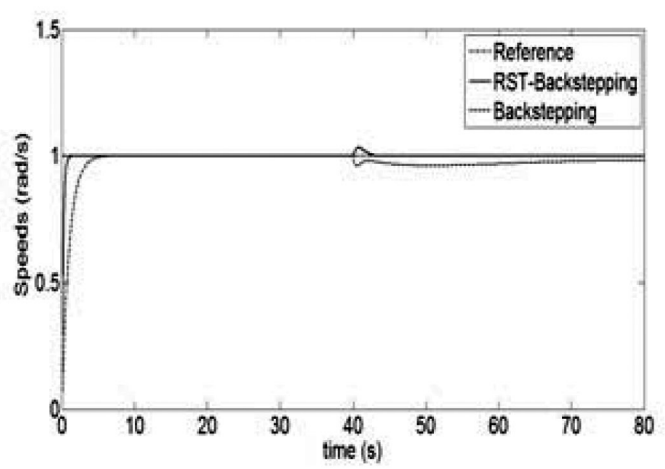

(c) Speeds $v_{2}$

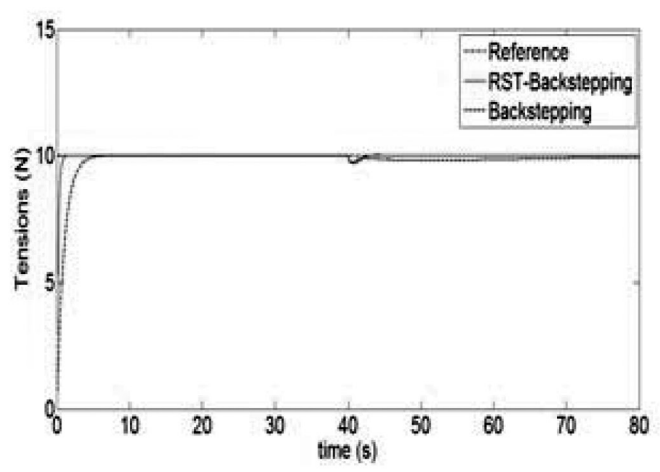

(e) Tensions $t_{2}$

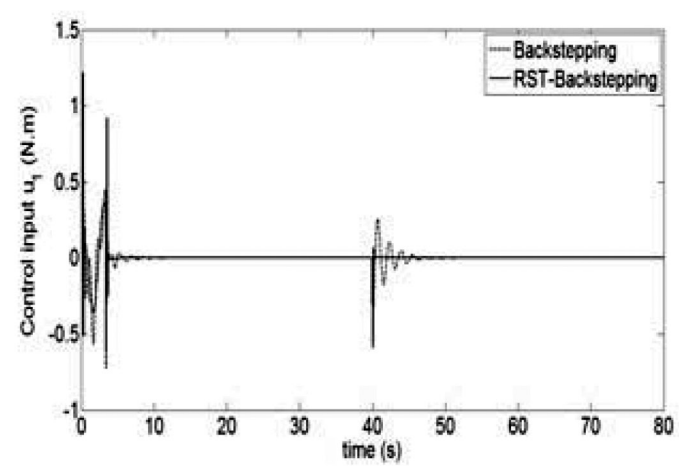

(b) Control inputs $u_{1}$

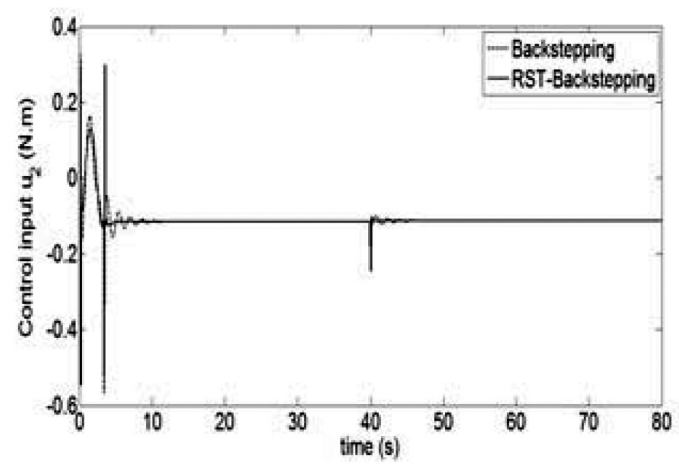

(d) Control inputs $u_{2}$

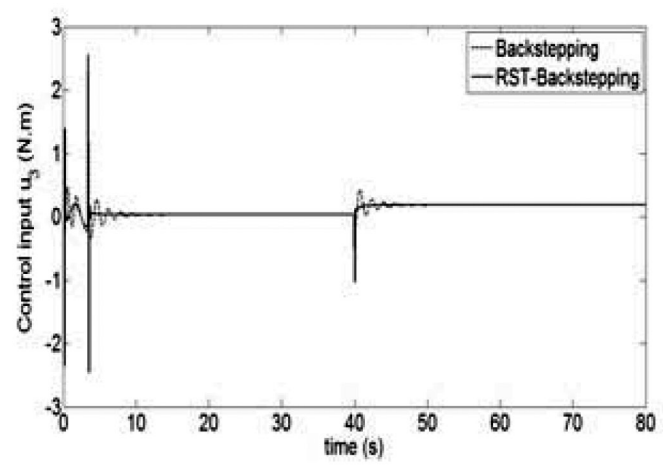

(f) Control inputs $u_{3}$

Fig. 5. Performance evaluation under the second scenario. (a) web tensions $t_{1}$, (b) control inputs $u_{1}$, (c) web velocities $V_{2}$, (d) control inputs $u_{2}$, (e) web tensions $t_{2}$, (f) control inputs $u_{3}$.

To summarise, the designed FTC method has better performance for fault compensation of the web-winding system when the system suffers from sensor fault and uncertainty.

\section{Conclusion}

In this paper, the new version of backstepping for FTC problem in case of presence disturbance, uncertainty, and sensor fault has been proposed and analysed for real industrial web-winding systems. An effective control 
strategy is proposed by means of the combined RST-backstepping method to ensure the rejection of any type of considered perturbations. The condition of asymptotic stability of the closed-loop system is proved by Lyapunov theorem. Finally, simulations results display a clear improvement in robustness and performance, this improvement is palpable in the quality of the signal, and the level of rejection of the affected perturbations confirm that the suggested technique not only rejects the disturbances and uncertainty but also guarantees that the system outputs can track the reference values in the presence of the considered faults. Future research will address the optimum design of the suggested control.

\section{References}

Ali, Z. A., Wang, D-B., Javed, R. and Akbar, A. (2016). Modeling \& Controlling the Dynamics of Tri-rotor UAV Using Robust RST Controller with MRAC Adaptive Algorithm. International Journal of Control and Automation, 9(3), pp. 61-76.

Arsalan, M., Iftikhar, R., Ahmad, I., Hasan, A., Sabahat, K. and Javeria, A. (2018). MPPT for Photovoltaic System Using Nonlinear Backstepping Controller with Integral Action. Solar Energy, 170, pp. 192-200.

Bodó, Z. and Lantos, B. (2019). Integrating Backstepping Control of Outdoor Quadrotor UAVS. Periodica Polytechnica Electrical Engineering and Computer Science, 63(2), pp. 122-132.

Brahim, M., Bahri, I. and Bernard, Y. (2017). Real Time Implementation of $\mathrm{H}$-infinity and RST Motion Control of Rotary Traveling Wave Ultrasonic Motor. Mechatronics, 44, pp. 14-23.

Chen, CX., Xie, YX. and Lan, YH. (2015). Backstepping Control of Speed Sensorless Permanent Magnet Synchronous Motor Based on Slide Model Observer. International Journal of Automation and Computing, 12(2), pp. 149-155.

Chu, X. Nian, X. Wang, H. and Xiong, H. (2019). Distributed Fault Tolerant Tracking Control for Large-Scale Multi-motor Web-winding Systems. IET Control Theory \& Applications, 13(4), pp. 543-553.

Dashkovskiy, S. N. and Pavlichkov, S. S. (2018). Integrator Backstepping for Uncertain Nonlinear Systems with Non-Smooth Dynamics. European Journal of Control, 40, pp. 68-79.

Elbakri, A. and Boumhidi, I. (2020). A New Intelligent Fault-tolerant Control Scheme for Wind Energy Systems Under Actuator Faults. European Journal of Electrical Engineering, 22(3), pp. 265-272.

Elmahfoud, M., Bossoufi, B., Taoussi, M., Elouanjli, N. and Derouich, A. (2020). Comparative Study Between Backstepping Adaptive and Field Oriented Controls for Doubly Fed Induction Motor. European Journal of Electrical Engineering, 22(3), pp. 209-221.
Haouari, F. O., Bali, N., Tadjine, M. O. and Boucherit, M. S. (2018). An Observer for Magnetic Levitation Control Based on a Coefficient Diagram Method and Backstepping. Archives on Electrical Engineering, 67(2), pp. 403-417.

Haouari, F., Gouri, R., Bali, N., Tadjine, M. and Boucherit, M. S. (2019). An Artificial Bee Optimization Based on Command Filtered CDM-Backstepping For Electro-Pneumatic System. Periodica Polytechnica Electrical Engineering and Computer Science, 63(3), pp. 235-241.

Haouari, F., Gouri, R., Bali, N., Tadjine, M. and Boucherit, M. S., (2020). Performance Enhancement of Electrohydraulic Servo System Using Teaching Learning-Based Optimization and CDMBackstepping with Disturbance Observer. Journal of Control Automation and Electrical Systems, 31(4), pp. 926-934.

Herizi, O. and Barkat, S. (2019). Backstepping Control Associated to Modified Space Vector Modulation for Quasi Z-Source Inverter Fed by a PEMFC. European Journal of Electrical Engineering, 21(2), pp. 125-132.

Hou, H., Nian, X., Xiong, H., Wang, Z. and Peng, Z. (2016). Robust Decentralized Coordinated Control of a Multimotor Web-Winding System. IEEE Transactions on Control Systems Technology, 24(4), pp. 1495-1503.

Jiang, T., Lin, D. and Song, T. (2018). Finite-Time Backstepping Control for Quadrotors with Disturbances and Input Constraints. IEEE Access, 6, pp. 62037-62049.

Karabacak, M. and Eskikurt, H. I. (2011). Speed and Current Regulation of a Permanent Magnet Synchronous Motor via Nonlinear and Adaptive Backstepping Control. Mathematical and Computer Modelling, 53(9-10), pp. 2015-2030.

Khadraoui, S., Nounou, H. N., Nounou, M. N., Datta, A. and Bhattacharyya, S. P. (2014). A Measurementbased Technique for Designing Fixed-order RST Controllers and Application to a Coupled 
Water Tank System. Systems Science \& Control Engineering, 2(1), pp. 484-492.

Klimkowski, K. (2017). Compensation Methods of Current and Speed Sensor Faults for a Vector Controlled Induction Motor Drive System. Power Electronics and Drives, 2(37), pp. 1.

Klimkowski, K. (2016). An Artificial Neural Networks Approach to Stator Current Sensor Faults Detection for DTC-SVM Structure. Power Electronics and Drives, 1(36), pp. 1.

Li, C., Zhang, Y. and Li, P. (2017). Full Control of a Quadrotor Using Parameter-scheduled Backstepping Method: Implementation and Experimental Tests. Nonlinear Dynamic, 89(2), pp. 1259-1278.

Liu, Y., Liu, X., Jing, Y. and Zhou, S. (2018). Adaptive Backstepping $\mathrm{H}_{\infty}$ Tracking Control with Prescribed Performance for Internet Congestion. ISA Transactions, 72, pp. 92-99.

Medjmadj, S. (2019). Fault Tolerant Control of PMSM Drive Using Luenberger and Adaptive Backemf Observers. European Journal of Electrical Engineering, 21(3), pp. 333-339.

Mohamed, H., Abdelmadjid, B. and Lotfi, B. (2020). Improvement of Direct Torque Control
Performances for Induction Machine Using a Robust Backstepping Controller and a New Stator Resistance Compensator. European Journal of Electrical Engineering, 22(2), pp. 137-144.

Ponsart, J. C., Theilliol, D. and Aubrun, C. (2010). Virtual Sensors Design for Active Fault Tolerant Control System Applied to a Winding Machine. Control Engineering Practice, 18(9), pp.1037-1044.

Pagilla, P. R., Siraskar, N. B. and Dwivendula, R. V. (2007). Decentralized Control of Web Processing Lines. IEEE Transactions on Control Systems Technology, 15(1), pp. 106-117.

Rodrigues M., Sahnoun M., Theilliol D. and Ponsart J. C. (2013). Sensor Fault Detection and Isolation Filter for Polytopic LPV Systems: A Winding Machine Application. Journal of Process Control, 23(6): 805-816.

Xiao, Y., Huang, S., Chen, H. and Xu, B. (2011). Research on Tension Control Strategy of Battery Pole-piece Winding and Unwinding System Based on Fault-tolerant Control. Procedia Engineering, 15(1), pp. 5073-5078.

Yu, J., Shi, P. and Zhao, L. (2018). Finite-Time Command Filtered Backstepping Control for a Class of Nonlinear Systems. Automatica, 92, pp. 173-180. 